\title{
Window of Opportunity: Postpartum Screening of Women with Gestational Diabetes for Early Detection of Prediabetes and Type 2 Diabetes
}

\author{
Cassandra E. Henderson, Jan Kavookjian, Harris Leitstein, June M. McKoy, Wambui Jane Murage \\ and Ruth D. Lipman*
}

American Association of Diabetes Educators, 200 W. Madison Street, Suite 800, Chicago, Il 60606, USA

\begin{abstract}
Gestational diabetes is a condition characterized by glucose intolerance during pregnancy, with defined approaches for screening, treatment, and follow-up. It is associated with a variety of adverse birth outcomes, including excessive fetal weight gain and related increases in the rate of cesarean delivery and perinatal injury as well as increased risk for developing type 2 diabetes for women who have had gestational diabetes. A diagnosis of gestational diabetes may also be a manifestation of pre-existing type 2 diabetes. Nonetheless, a substantial proportion of women with a history of gestational diabetes fail to receive the recommended postpartum glucose screening. This failure to conduct follow-up screening of women with gestational diabetes after delivery represents a missed opportunity for earlier diagnosis of diabetes, and chance to increase the awareness of women of their future risk for developing diabetes. This paper explores the barriers contributing to the lack in follow-up screening and makes recommendations about addressing these problems.
\end{abstract}

Keywords: Barriers, gestational diabetes, screening, type 2 diabetes.

\section{BACKGROUND}

Gestational diabetes mellitus (GDM) is defined as carbohydrate intolerance that begins or is first recognized during pregnancy [1]. This diagnostic criterion for GDM was established nearly 50 years ago [2]. In the intervening years, much has been learned about how the physiological changes associated with pregnancy affect insulin resistance and pancreatic $\beta$-cell defect [3]. It has long been postulated that placental hormone production and increased cortisol were solely responsible for the decreased insulin sensitivity observed during pregnancy [4]. The spectrum of contributing factors is now understood to likely include changes to circulating levels of adipokines such as adiponectin (a protein involved in glucose regulation and the metabolism of fat for energy production), as well as inflammatory mediators produced by the placenta, such as TNF- $\alpha$ and IL6; these may contribute to the insulin resistance that occurs in pregnancy [5].

However, casting gestational diabetes merely as a pregnancy associated complication that mostly resolves after delivery $[6,7]$, is inappropriate in an era where the prevalence of the metabolic syndrome and diabetes itself is increasing in all demographics, including that of women of childbearing age $[8,9]$. Reflecting this trend, the Standards of Medical Care in Diabetes 2012 not only calls for women with risk factors to be screened for undiagnosed type 2 diabetes at the first prenatal visit, but also defines gestational diabetes as 'diabetes diagnosed during pregnancy that is not clearly overt diabetes [10]; this represents additional

Address correspondence to this author at the American Association of Diabetes Educators, 200 W. Madison Street, Suite 800, Chicago, Il 60606, USA; Tel: 312-601-4854; Fax: 312-424-2426;

E-mail: rlipman@aadenet.org precision in the definition of GDM, which as recently as 2009 was defined simply as 'diabetes diagnosed during pregnancy [6].

The consensus recommendation stemming from the multi-stakeholder meetings held in 2008-2009 by the International Association of Diabetes and Pregnancy Study Group is that all women not known to have diabetes should undergo screening using a 75 -g oral glucose tolerance test (OCTT) at 24-28 weeks of gestation; this should be performed in the morning, after an overnight fast of at least 8 hours, to measure plasma glucose levels at fasting and at 1 and 2 hours. An abnormal value for any of the three diagnostic cut point (Table 1) measurements is sufficient to make the diagnosis of GDM [11]. The cut points for these abnormal values convey an odds ratio for adverse outcomes of at least 1.75 [11].

Table 1. Abnormal Glucose Concentration Threshold

\begin{tabular}{|c|c|c|}
\hline Glucose Measure & $\mathbf{m g} / \mathbf{d l}$ & $\mathbf{n m o l} / \mathbf{l}$ \\
\hline \hline Fasting plasma glucose & $\geq 92$ & $\geq 5.1$ \\
\hline 1-h plasma glucose & $\geq 180$ & $\geq 10.0$ \\
\hline 2-h plasma glucose & $\geq 153$ & $\geq 8.5$ \\
\hline
\end{tabular}

One focus of GDM related research is GDM as a risk factor for development of type 2 diabetes (T2D). A systematic review of the risk of T2D after GDM found that nearly $60 \%$ of women giving birth who had GDM during pregnancy will develop T2D within 10 years of delivery [12]. To address this risk, the Centers for Disease Control (CDC), the American College of Obstetrics and Gynecology (ACOG), and the American Diabetes Association (ADA) 
have recommended protocols that include regular postpartum glucose screening and the use of lifestyle changes and/or pharmacotherapy to prevent or delay the onset of T2D [13]. Nevertheless, although these advisories are widely accepted, the guidelines are often not followed. Indeed, in one report only $34 \%$ to $74 \%$ of women with a history of GDM received the recommended postpartum glucose screening [14]. These missed glucose screening opportunities and lack of effective intervention may be associated with the marked increase seen in the incidence of T2D among women of childbearing age $[8,9]$. For this cohort of women, pregnancy may represent the first time that they undergo screening for diabetes. Thus, having glucose intolerance detected during pregnancy does not exclude the possibility that onset may have been prior to conception [15]. Rather than gestational diabetes, their diabetes may more accurately represent discovery of preexisting glucose intolerance [16].

For women who may have subsequent pregnancies, there is potential for developing T2D due to considerable secretory demands posed by the physiologic insulin resistance of a subsequent pregnancy [17]. However, some studies have found that subsequent pregnancy is not necessarily associated with an increased risk of T2D [18]. As the data are not definitive and because GDM is associated with increased risk of shoulder dystocia, preeclampsia, and macrosomia [19], insight into their history of GDM and possible risk of perinatal complications might influence their decisions regarding nutrition and activity for future pregnancies.

It is estimated that as many as $30 \%$ of those diagnosed with GDM have impaired glucose regulation [20]. Harm can occur even while T2D is asymptomatic; this is important since duration of hyperglycemia is a predictor of adverse outcomes [21]. It is sobering to note that the prevalence of retinopathy is found in upwards of nearly $8 \%$ of those participating in a population based screen for diabetes [22] and as high as $20 \%$ in some populations [23]. Kidney damage has also been found to antecede the diagnosis of diabetes, with approximately $42 \%$ of those determined to have diabetes in the NHANES data also having attendant chronic kidney disease [24]. Failure to undergo postpartum screening for women with diagnosed diabetes during the perinatal period occurs in a readily identifiable group of individuals with potentially undiagnosed T2D.

We suggest that failure to routinize performance of the recommended postpartum glucose screen is a detriment to public health. Barriers to women being screened after a GDM affected pregnancy have been broadly attributed to three categories of stakeholders, namely patients, providers, and systems.

\section{PATIENT RELATED BARRIERS}

A woman may not be aware that 1) GDM increases her risk for developing T2D and/or 2) that there are proven methods to prevent or delay development of the disease; women in this cohort who do not have postpartum screening report being inadequately informed and failed to perceive themselves at risk [25]. One study found screening rates improved from $14.3 \%$ to $51 \%-60 \%$ when the patient received education as well as follow-up reminders [26].
Patients have identified lack of time as a primary barrier [27], with most of their time expended on attending to the needs of their newborn and their other children [28]. Women also reported fear of receiving bad news, as well as a less than satisfactory experience with medical care and service as factors contributing to their choice not to pursue screening after delivery [28]. Additionally, social/economic or cultural barriers are believed to play a role in pursue or decline GDM follow-up. Furthermore, discrepant screening rates were reported for various racial and ethnic groups [29]. The postpartum glucose screening rate of $27 \%$ for African American women was lower than that for Hispanic $(51 \%)$ and Caucasian (48\%) women.

\section{PROVIDER RELATED BARRIERS}

The latest guidelines for GDM screening [11] were developed by the International Association of Diabetes and Pregnancy Study Group (IADPSG), and are based in large part on findings from the Hyperglycemia and Adverse Pregnancy Outcomes (HAPO) study [30]. The HAPO study followed approximately 23,000 women who had an oral glucose tolerance test administered between 24 and 32 weeks gestation. In this multicenter, multinational study, investigators found a progressive rise in the primary outcomes of high birth weight, cesarean delivery, fetal $\mathrm{C}$ peptide levels, and neonatal hypoglycemia associated with increasing maternal blood glucose. There were similar increases in the rate of secondary outcomes such as shoulder dystocia, need for intensive neonatal care, and preeclampsia. Interestingly, there was no glycemic threshold for increased risk.

However, not all professional groups, including the American Congress of Obstetricians and Gynecologists [11], have endorsed these new diagnostic criteria. Concerns have been raised about the healthcare costs associated with the significant change in practice as well as possible harms that might result from identifying more women with GDM. The ADA considers these new diagnostic criteria "medicalization" of what would previously have been categorized as a normal pregnancy [10]. Undoubtedly, this lack of consensus leads to confusion regarding a pregnant woman's clinical status, treatment plan, and ultimately clinical follow-up.

Layered on top of the controversy related to appropriate thresholds for defining GDM, are the issues posed by the inconsistent screening guidelines for women following GDM itself. This inconsistency can lead to provider confusion and inaction [31]. Postpartum screening intervals vary across guidelines from six weeks to up to one year after delivery. The ADA indicates that women with a history of GDM should be screened for diabetes at 6-12 weeks postpartum, using non-pregnant OGTT criteria [10]. However, as this may represent the final interaction with the $\mathrm{OB} / \mathrm{GYN}$ provider, even if women are screened at this time point, the results may not be communicated.

In addition to the lack of consensus about the timing for postpartum screening, there is also lack of agreement about test to be used. Suggested screening methods include fasting plasma glucose (FPG) with or without a 75-gram 2-hour OGTT, and a 100 gram 3-hour OGTT. The OGTT is more sensitive than the FPG alone, with detection rates up to 
$100 \%$ versus $67 \%$ respectively [32]. Although Hemoglobin $\mathrm{A} 1 \mathrm{C}$ has been suggested as an appropriate method for general screening for diabetes [32], and is a readily available assay, it is not recommended at the postpartum visit for women who received treatment for hyperglycemia during pregnancy because it remains to be determined whether their results reflect their current ability to regulate circulating glucose [10].

Late provider transfer or provider co-management of prenatal care often leads to a lack of ownership of patient follow up responsibility [32]. Obstetric providers (OB) are more likely to ask about and be aware of a woman's GDM history, but are less likely than other primary care providers (PCPs) to order postpartum testing where there is an established history of GDM. Conversely, while PCPs are more likely than OB providers to order postpartum testing for a known GDM history, they are less likely to ask about a past history of GDM [33].

\section{SYSTEMS RELATED BARRIERS}

A frequently identified systems barrier to early diabetes intervention is lacking the means to identify persons fitting the appropriate risk profile [34]. Women with a diagnosis of GDM during pregnancy, however, have already been identified as representing a cohort at risk for diabetes. Nonetheless, communication needed for optimal transition of care between obstetric and other primary care providers is often poor and therefore represents a missed screening opportunity [35].

Electronic medical record (EMR) systems could be a means of eliminating communication gap between providers by documenting and flagging/prompting screenings. Such systems, however, are only as good as the information entered. At least one study has found that history of GDM was documented for less than half the patients in whom it had been diagnosed [35]. Furthermore, implementation and utilization of EMR remains a challenge in smaller practices [35].

\section{RECOMMENDATIONS}

Recommendations for T2D screening and preventing future complications include the following:

- Create a campaign to develop simple, culturally sensitive patient education material about the health significance of having had GDM. Raising awareness can affect a woman's health beliefs, including perceived susceptibility, which has been shown with mammography and colonoscopy to increase health screening rates.

- $\quad$ Solicit added influence from the woman's support network, expanding this educational campaign to target individuals who are friends/spouses, clergy or other family members of women who had a pregnancy complicated by GDM.

- Incorporate relevant education material into prenatal classes and disseminate it in OB patient waiting areas and hospital admission/discharge delivery packets. Inclusion of this information in such locations may prompt women to query their physicians, thus potentially impacting provider barriers to screening.
- $\quad$ Conduct appropriately powered prospective studies of women with a history of GDM to evaluate the clinical efficacy of hemoglobin A1C as a screen for T2D. If efficacious, use of $\mathrm{A} 1 \mathrm{C}$ as a standard postpartum screening test would decrease the current uncertainty regarding preferred screening method. It would obviate pre-testing preparation requirements, thus addressing patient barriers of time pressures and/or preparation aversion.

- Harness the potential of EMR to enhance communication between multiple providers relative to the diagnosis of GDM, to maximize postpartum screening through utilization of reminder prompts, and to improve overall continuity of care.

\section{CONCLUSION}

Women with gestational diabetes mellitus (GDM) should be monitored post-partum to exclude ongoing diabetes and to decrease risk factors associated with subsequent development of type 2 diabetes. The GDM patient cohort is unique in that they have previously engaged with the healthcare system about diabetes care, but have either failed to adhere to or have not been provided continued monitoring post-delivery. Failure on the part of healthcare providers to screen for diabetes in women who have had GDM or to encourage patient adherence through diabetes education is a missed opportunity to heighten patient awareness about their health risks and to facilitate the development of healthy lifestyles and healthcare strategies to prevent or delay development of this chronic disease. Further studies to identify modifiable factors that converge to prevent continued assessment and care for women with GDM beyond the post-partum period are needed.

\section{ACKNOWLEDGEMENTS}

This manuscript is the work of the American Association of Diabetes Educators 2012 Practice and Research Papers workgroup.

\section{CONFLICT OF INTEREST}

The authors confirm that this article content has no conflicts of interest.

\section{REFERENCES}

[1] ACOG Practice Bulletin. Clinical management guidelines for obstetrician-gynecologists. Number 30, September 2001 (replaces Technical Bulletin Number 200, December 1994). Gestational diabetes. Obstet Gynecol 2001; 98(3): 525-38.

[2] O'Sullivan JB, Mahan CM. Criteria for the oral glucose tolerance test in pregnancy. Diabetes 1964; 13: 278-85.

[3] Kaaja R, Ronnemaa T. Gestational diabetes: pathogenesis and consequences to mother and offspring. Rev Diabet Stud 2008; 5(4): 194-202.

[4] Catalano PM. Obesity, insulin resistance, and pregnancy outcome. Reproduction 2010; 140(3): 365-71.

[5] Vrachnis N, Belitsos P, Sifakis S, et al. Role of adipokines and other inflammatory mediators in gestational diabetes mellitus and previous gestational diabetes mellitus. Int J Endocrinol 2012; 2012: 549748 .

[6] Standards of medical care in diabetes--2009. Diabetes Care 2009; 32 (Suppl 1): S13-61.

[7] Damm P. Gestational diabetes mellitus and subsequent development of overt diabetes mellitus. Dan Med Bull 1998; 45(5): 495-509.

[8] Ford ES, Giles WH, Dietz WH. Prevalence of the metabolic syndrome among US adults: findings from the third National 
Health and Nutrition Examination Survey. JAMA 2002; 287(3): 356-9.

[9] Ramos RG, Olden K. The prevalence of metabolic syndrome among US women of childbearing age. Am J Public Health 2008; 98(6): 1122-7.

[10] Standards of medical care in diabetes--2012. Diabetes Care 2012;35 (Suppl 1): S11-63.

[11] Metzger BE, Gabbe SG, Persson B, et al. International association of diabetes and pregnancy study groups recommendations on the diagnosis and classification of hyperglycemia in pregnancy. Diabetes Care 2010; 33(3): 676-82.

[12] Kim C, Newton KM, Knopp RH. Gestational diabetes and the incidence of type 2 diabetes: a systematic review. Diabetes Care 2002; 25(10): 1862-8.

[13] Ratner RE, Christophi CA, Metzger BE, et al. Prevention of diabetes in women with a history of gestational diabetes: effects of metformin and lifestyle interventions. J Clin Endocrinol Metab 2008; 93(12): 4774-9.

[14] Tovar A, Chasan-Taber L, Eggleston E, Oken E. Postpartum screening for diabetes among women with a history of gestational diabetes mellitus. Prev Chronic Dis. 2011; 8(6): A124.

[15] Diagnosis and classification of diabetes mellitus. Diabetes Care 2011; 34 (Suppl 1): S62-9.

[16] Harris MI. Gestational diabetes may represent discovery of preexisting glucose intolerance. Diabetes Care 1988; 11(5): 402-11.

[17] Retnakaran R, Shah BR. Abnormal screening glucose challenge test in pregnancy and future risk of diabetes in young women. Diabet Med 2009; 26(5): 474-7.

[18] Retnakaran R, Austin PC, Shah BR. Effect of subsequent pregnancies on the risk of developing diabetes following a first pregnancy complicated by gestational diabetes: a population-based study. Diabet Med 2011;28(3): 287-92.

[19] Evensen AE. Update on gestational diabetes mellitus. Prim Care 2012; 39(1): 83-94.

[20] Lawrence JM, Black MH, Hsu JW, Chen W, Sacks DA. Prevalence and timing of postpartum glucose testing and sustained glucose dysregulation after gestational diabetes mellitus. Diabetes Care 2010;33(3): 569-76.

[21] Charfen MA, Ipp E, Kaji AH, Saleh T, Qazi MF, Lewis RJ. Detection of undiagnosed diabetes and prediabetic states in highrisk emergency department patients. Acad Emerg Med 2009; 16(5): 394-402.

[22] Spijkerman AM, Dekker JM, Nijpels G, et al. Microvascular complications at time of diagnosis of type 2 diabetes are similar among diabetic patients detected by targeted screening and patients newly diagnosed in general practice: the hoorn screening study. Diabetes Care 2003; 26(9): 2604-8.

[23] Harris MI, Klein R, Welborn TA, Knuiman MW. Onset of NIDDM occurs at least 4-7 yr before clinical diagnosis. Diabetes Care 1992; 15(7): 815-9.

[24] Plantinga LC, Crews DC, Coresh J, et al. Prevalence of chronic kidney disease in US adults with undiagnosed diabetes or prediabetes. Clin J Am Soc Nephrol 2010; 5(4): 673-82.

[25] Kim C, McEwen LN, Piette JD, Goewey J, Ferrara A, Walker EA. Risk perception for diabetes among women with histories of gestational diabetes mellitus. Diabetes Care 2007; 30(9): 2281-6.

[26] Clark HD, Graham ID, Karovitch A, Keely EJ. Do postal reminders increase postpartum screening of diabetes mellitus in women with gestational diabetes mellitus? A randomized controlled trial. Am J Obstet Gynecol 2009; 200(6): 634 e1-7.

[27] Keely E, Clark H, Karovitch A, Graham I. Screening for type 2 diabetes following gestational diabetes: family physician and patient perspectives. Can Fam Physician 2010; 56(6): 558-63.

[28] Bennett WL, Ennen CS, Carrese JA, et al. Barriers to and facilitators of postpartum follow-up care in women with recent gestational diabetes mellitus: a qualitative study. J Womens Health (Larchmt) 2011; 20(2): 239-45.

[29] Ferrara A, Peng T, Kim C. Trends in postpartum diabetes screening and subsequent diabetes and impaired fasting glucose among women with histories of gestational diabetes mellitus: A report from the Translating Research Into Action for Diabetes (TRIAD) Study. Diabetes Care 2009; 32(2): 269-74.

[30] Metzger BE, Lowe LP, Dyer AR, et al. Hyperglycemia and adverse pregnancy outcomes. N Engl J Med 2008; 358(19): 1991-2002.

[31] Baker AM, Brody SC, Salisbury K, Schectman R, Hartmann KE. Postpartum glucose tolerance screening in women with gestational diabetes in the state of North Carolina. N C Med J 2009; 70(1): 149 .

[32] Blatt AJ, Nakamoto JM, Kaufman HW. Gaps in diabetes screening during pregnancy and postpartum. Obstet Gynecol 2011; 117(1): 61-8.

[33] Shah BR, Lipscombe LL, Feig DS, Lowe JM. Missed opportunities for type 2 diabetes testing following gestational diabetes: a population-based cohort study. BJOG 2011;118(12): 1484-90.

[34] Green LW, Brancati FL, Albright A. Primary prevention of type 2 diabetes: integrative public health and primary care opportunities, challenges and strategies. Fam Pract 2012; 29 (Suppl 1): i13-23.

[35] Stuebe A, Ecker J, Bates DW, Zera C, Bentley-Lewis R, Seely E. Barriers to follow-up for women with a history of gestational diabetes. Am J Perinatol 2010; 27(9): 705-10. 